\title{
Recent advances in the construction of nanozyme-based logic gates
}

\author{
Fang $\mathrm{Pu}^{1}$, Jinsong $\operatorname{Ren}^{1,2}$, Xiaogang $\mathrm{Qu}^{1,2}$ \\ ${ }^{1}$ Laboratory of Chemical Biology and State Key Laboratory of Rare Earth Resource Utilization, Changchun Institute \\ of Applied Chemistry, Chinese Academy of Sciences, Changchun 130022, China \\ ${ }^{2}$ University of Science and Technology of China, Hefei 230026, China
}

Received: 28 June 2020 / Accepted: 15 October 2020 / Published online: 21 November 2020

\begin{abstract}
Nanozymes, nanomaterials with enzyme-like activity, have been considered as promising alternatives of natural enzymes. Molecular logic gates, which can simulate the function of the basic unit of an electronic computer, perform Boolean logic operation in response to chemical, biological, or optical signals. Recently, the combination of nanozymes and logic gates enabled bioinformation processing in a logically controllable way. In the review, recent progress in the construction of nanozyme-based logic gates integrated with their utility in sensing is introduced. Furthermore, the issues and challenges in the construction processes are discussed. It is expected the review will facilitate a comprehensive understanding of nanozyme-based logic systems.
\end{abstract}

Keywords Nanozyme, Logic gate, Sensor, Regulation, Input

\section{INTRODUCTION}

The computer processes digital information and performs logical operation based on silicon-circuitry and logic gates. Electrical signals are used as inputs and outputs of logic gates which follow the laws of Boolean algebra. The absence and presence of electrical signals are defined as 0 and 1, respectively. Boolean logic gates produce binary outputs $0 / 1$ upon treatment with binary inputs $0 / 1$, corresponding to AND, OR, INHIBIT, NAND, NOR, XOR, XNOR, etc. Originating from the requirements of computer miniaturization and increase of function density, Boolean logic operations have been realized on the molecular levels (Andreasson and Pischel 2010; de Silva and McClenaghan 2004; Erbas-Cakmak et al. 2018; $\mathrm{Pu}$ et al. 2014b). In general, molecular logic gates are implemented based on molecular switching or chemical reaction. Various chemical and physical signals serve as inputs and as outputs. In the past few decades, inspired by the biocatalytic reactions in biological process, great

$\bowtie$ Correspondence: xqu@ciac.ac.cn (X. Qu) efforts have been made in construction of logic gates and computing components based on natural enzymes (Katz and Privman 2010; Lin et al. 2012). Nanozyme, the nanomaterial with enzyme-like properties, has attracted great attention in recent years (Huang et al. 2019; Wei and Wang 2013; Wu et al. 2019a). Compared with natural enzyme, nanozyme presented the advantages of easy preparation and modification, high stability, duration under harsh conditions, size/morphology/composition dependent activity, and low cost. As an alternative, nanozymes show potential application in environment, biomedicine, and food industry (Huang et al. 2018, 2019; Wang et al. 2016). Nanozymes have also been used as computing elements to mimic the functions of logic gates. On the one hand, nanozyme-based logic gates borrowed some ideas from natural enzyme-based biological information processing. On the other hand, the properties of nanomaterials themselves made their performance superior to that of natural enzymes. However, so far, there is no review on the nanozyme-based logic gates. In this review, we illustrated how Boolean logic gates were realized using 
nanozymes. Meanwhile, the applications of nanozymebased logic gate in sensing were introduced. Furthermore, the existing issues and challenges in the field were discussed.

\section{TYPES OF NANOZYMES USED FOR CONSTRUCTION OF LOGIC GATES}

Current studies on logic gates focus on oxidoreductase, including oxidase, peroxidase, and catalase (Table 1). Oxidase catalyzes the oxidation of a substrate with the assistance of $\mathrm{O}_{2}$ to produce oxidized product and $\mathrm{H}_{2} \mathrm{O}$ / $\mathrm{H}_{2} \mathrm{O}_{2} / \mathrm{O}_{2}^{-}$. Glucose oxidase can catalyze glucose to yield gluconic acid and $\mathrm{H}_{2} \mathrm{O}_{2}$ in the presence of $\mathrm{O}_{2}$. Gold nanoparticles (AuNPs) are the most studied mimic of glucose oxidase (Zhang et al. 2018). Peroxidase can oxidize a substrate with the help of $\mathrm{H}_{2} \mathrm{O}_{2}$. Carbon-based nanomaterials (graphene, carbon nanotubes, etc.) and metal oxides $\left(\mathrm{Fe}_{3} \mathrm{O}_{4}, \mathrm{CuO}, \mathrm{V}_{2} \mathrm{O}_{5}\right.$, etc.) have been found to possess excellent peroxidase-like properties (Andre et al. 2011; Gao et al. 2007; Song et al. 2010; Wang et al. 2018b). Catalase decomposes $\mathrm{H}_{2} \mathrm{O}_{2}$ into $\mathrm{H}_{2} \mathrm{O}$ and oxygen. Some metal oxides were reported to present catalase-like activity (Mu et al. 2013). Moreover, some nanozymes possess multiple enzyme-like activities ( Wu et al. 2019a). For instance, AuNPs with the diameter of $13 \mathrm{~nm}$ have peroxidase-, oxidase-, and catalase-like activities. Taking advantage of these nanozymes, a series of logic gates were constructed.

\section{LOGIC GATES BASED ON NANOZYMES}

Different from conventional silicon-based digital elements which use electrical signals as inputs and outputs, the nature of the input and output signals of molecular logic gates is different. For nanozyme-based logic gates, nanozymes, substrates, external substances, and physical stimuli could serve as logic unit or inputs. The final absorbance or fluorescence of reaction products serves as outputs. In this review, according to the composition of logic gates, nanozyme-based logic gates are categorized into two classes: nanozymes themselves as inputs and regulation factors of nanozymes as inputs.

\section{Nanozymes as inputs to construct logic gates}

Inspired by intracellular enzyme-participated redox balance system, Huang et al. constructed an AND gate by employing two artificial enzymes: $\mathrm{Au}$ nanoparticledoped mesoporous silica nanoparticles $\left(\mathrm{SiO}_{2}-\mathrm{Au}\right)$ and G-quadruplex-hemin DNAzyme as inputs (Huang et al. 2017). $\mathrm{SiO}_{2}-\mathrm{Au}$ with glucose oxidase-like activity could catalyze glucose to generate gluconic acid and $\mathrm{H}_{2} \mathrm{O}_{2}$. DNAzyme presented peroxidase-like activity, through which $\mathrm{H}_{2} \mathrm{O}_{2}$ could be transformed to $\cdot \mathrm{OH}$ and ABTS could be oxidized to $\mathrm{ABTS}^{+}$. The cascade reaction produced colored product when both the inputs existed, corresponding to AND gate (Fig. 1). Both G-quadruplex-hemin DNAzyme and $\mathrm{V}_{2} \mathrm{O}_{5}$ nanowire could mimic peroxidase to catalyze the same reaction.

Table 1 The representative materials for construction of logic gates

\begin{tabular}{|c|c|c|}
\hline Enzyme-like activity & Nanozymes & References \\
\hline \multirow[t]{2}{*}{ Glucose oxidase } & $\mathrm{Au}$ nanoparticle-doped mesoporous silica nanoparticles $\left(\mathrm{SiO}_{2}-\mathrm{Au}\right)$ & Huang et al. (2017) \\
\hline & AuNPs & Zhan et al. (2014) \\
\hline \multirow[t]{8}{*}{ Peroxidase } & $\mathrm{V}_{2} \mathrm{O}_{5}$ nanowire & Huang et al. (2017) \\
\hline & CuO:Graphene nanosphere (CuO:GNS) & Sharma and Mobin (2017) \\
\hline & AuNPs & Lien et al. $(2013,2014)$ \\
\hline & bimetallic nanoparticle PtAuNPs & Lin et al. (2017) \\
\hline & Hemin-graphene (HG) hybrid nanosheet & Lin et al. (2014) \\
\hline & Copper (II)-based metal-organic frameworks (Cu-MOFs) & Wang et al. (2018a) \\
\hline & histidine-protected gold nanoclusters (His-AuNCs) & Chen et al. (2019) \\
\hline & $\mathrm{NH}_{2}$-MIL-101 MOFs & Li et al. (2019) \\
\hline \multirow[t]{2}{*}{ Catalase } & AuNPs & Lien et al. (2013) \\
\hline & $\mathrm{Co}_{3} \mathrm{O}_{4}$ nanoparticles & Han et al. (2018) \\
\hline \multirow[t]{4}{*}{ Oxidase } & AuNPs & Lien et al. (2013) \\
\hline & Manganese dioxide nanosheet $\left(\mathrm{MnO}_{2} \mathrm{NS}\right)$ & Fan et al. (2017) \\
\hline & 9-mesityl-10-methylacridinium ion $\left(\mathrm{Acr}^{+}-\mathrm{Mes}\right)$ & Du et al. (2018) \\
\hline & $\mathrm{MnO}_{2}$ nanosheets & Zhao et al. (2018) \\
\hline
\end{tabular}




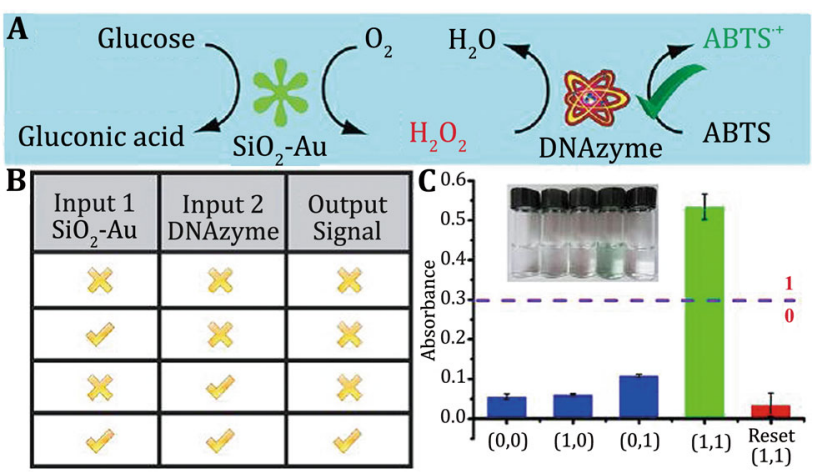

Fig. 1 Scheme (A) and truth table (B) of nanozyme-based AND gate. C Absorbance and photograph of reaction product after being treated with the combination of different inputs. Reproduced with permission of Wiley-VCH (Huang et al. 2017)

Based on it, an OR gate was constructed. $\mathrm{MnO}_{2}$ could catalyze the decomposition of $\mathrm{H}_{2} \mathrm{O}_{2}$ to produce $\mathrm{H}_{2} \mathrm{O}$ and $\mathrm{O}_{2}$. Without $\mathrm{H}_{2} \mathrm{O}_{2}$, DNAzyme could not transform ABTS to $\mathrm{ABTS}^{+}$. Combining $\mathrm{MnO}_{2}$ and DNAzyme, an INHIBIT gate was built.

Sharma et al. found that CuO:Graphene nanosphere (CuO:GNS) composite could mimic peroxidase (Sharma and Mobin 2017). Phenol coupled with 4-aminoantipyrine (4-AAP) in the presence of $\mathrm{H}_{2} \mathrm{O}_{2}$, the main product of oxidation of cholesterol by cholesterol oxidase, could be catalyzed by CuO:GNS to yield a red colored product. The reaction was employed for colorimetric sensing of $\mathrm{H}_{2} \mathrm{O}_{2}$ and cholesterol. Furthermore, using CuO:GNS and cholesterol as inputs, an AND gate was constructed.

\section{Logic systems based on the regulation of the catalytic activities of nanozymes}

Metal ions, biomolecules, $\mathrm{pH}$, and light illumination could regulate the catalytic activity of nanozymes. These external substances and physical stimuli were used as inputs to construct logic gates.

\section{Metal ions}

The catalytic activity of nanozyme can be activated or inhibited by depositing metal ions. Taking advantage of this property, Lien et al. constructed multiplex logic operations (OR, AND, INHIBIT, and XOR gates) through regulation of the enzyme-like activity of AuNPs upon addition of different metal ions (Lien et al. 2013). AuNPs $(13 \mathrm{~nm})$ exhibited multiple enzyme-like activities, including peroxidase-, oxidase-, and catalase-like activity. The oxidase-like activity of AuNPs could be enhanced by co-deposition of $\mathrm{Pt}^{4+} / \mathrm{Hg}^{2+}$, resulting from $\mathrm{Pt}^{4+} / \mathrm{Hg}^{2+}$-induced high oxidative catalytic ability and minimizing deactivation. An AND gate could be constructed using the oxidase-like activity of AuNPs and $\mathrm{Pt}^{4+} / \mathrm{Hg}^{2+}$ ions as inputs. The catalase-like activity of AuNPs could be enhanced by $\mathrm{Hg}^{2+}, \mathrm{Bi}^{3+}$, and their mixture, accompanied by stronger surface plasmon resonance (SPR) absorption. The catalase-like activity of AuNPs and $\mathrm{Hg}^{2+} / \mathrm{Bi}^{3+}$ as inputs was used to construct an OR gate. The peroxidase-like activity of AuNPs was very low due to the low variation of surface metal oxidation states. $\mathrm{Pb}^{2+}$ adsorbed on the surface enhanced the peroxidase-like activity, resulting from various valance (oxidation) states of the $\mathrm{Pb}\left(\mathrm{Pb}^{2+} / \mathrm{Pb}^{0}\right)$ and $\mathrm{Au}$ $\left(\mathrm{Au}^{+} / \mathrm{Au}^{0}\right) . \mathrm{Hg}^{2+}$ presented slight effect on the peroxidase-like activity due to its low oxidation state. The competition between $\mathrm{Pb}^{2+}$ and $\mathrm{Hg}^{2+}$ ions for AuNPs presented an INHIBIT logic operation, in which the peroxidase-like activity of AuNPs served as the output. The strong Au-Ag metallophilic interactions and ease of reduction of $\mathrm{Ag}^{+}$resulted in the enhanced peroxidaselike activity. In the presence of both $\mathrm{Ag}^{+}$and $\mathrm{Bi}^{3+}$, the formation of stable $\mathrm{Ag} / \mathrm{Bi}$ alloys decreased surface potentials and active sites, reducing the activity. The regulation of peroxidase-like activity of AuNPs by $\mathrm{Ag}^{+}$ and $\mathrm{Bi}^{3+}$ corresponded to a XOR gate. Furthermore, due to the stable surface lattice structures and/or low surface energy of $\mathrm{Pt}^{4+}$ and $\mathrm{Pb}^{2+}$, the peroxidase-like activity of AuNPs was low when one of them was presented. The co-deposition of $\mathrm{Pt}^{4+}$ and $\mathrm{Pb}^{2+}$ caused enhancement of the peroxidase-like activity. It might be attributed to that the formation of alloys with various valence states led to increased surface potentials and active sites. Based on it, the same group constructed AND gate using $\mathrm{Pt}^{4+} / \mathrm{Pb}^{2+}$ ions and the peroxidase-like activity of AuNPs in the presence of $\mathrm{S}_{2} \mathrm{O}_{3}^{2-}$ (Lien et al. 2014). Besides, enhanced activity could be achieved by $\mathrm{Bi}^{3+}$ due to the various valence (oxidation) states of $\mathrm{Bi}^{3+}$ and $\mathrm{Au}\left(\mathrm{Au}^{+} / \mathrm{Au}^{0}\right)$. Upon introduction of $\mathrm{Hg}^{2+}$, the surface of AuNPS was passivated, presented low activity. Thus, an INHIBIT gate using $\mathrm{Bi}^{3+} / \mathrm{Hg}^{2+}$ and peroxidaselike activity of AuNPs was constructed. An integrated logic circuit was also constructed for the visual detection of $\mathrm{Pb}^{2+}$ and $\mathrm{Hg}^{2+}$ (Fig. 2).

\section{Biomolecules}

Owing to easy synthesis and modification, robust physicochemical nature, tunable polymorphic structure, and specific recognition, nucleic acids are integrated with nanomaterials to construct logic gates $\mathrm{Pu}$ et al. 2014b). Recently, nucleic acids were used to manipulate catalytic behavior of nanozymes (Liu and Liu 2015; Zheng et al. 2011). However, the phenomenon and explanation for the regulation mechanism are 
Fig. 2 A Three-input/threeoutput integrated logic circuit. B Images of solutions of AuNPs $/ \mathrm{Bi}^{3+} / \mathrm{H}_{2} \mathrm{O}_{2}$ (upper) and AuNPs $/ \mathrm{Pt}^{4+} / \mathrm{S}_{2} \mathrm{O}_{3}^{2-} / \mathrm{H}_{2} \mathrm{O}_{2} /$ AR (bottom) treated with nothing (a), $\mathrm{Pb}^{2+}(\mathbf{b})$, $\mathrm{Hg}^{2+}$ (c), and $\mathrm{Pb}^{2+} / \mathrm{Hg}^{2+}$ (d). C Truth table. Reproduced with permission of American Chemical Society (Lien et al. 2014)
A

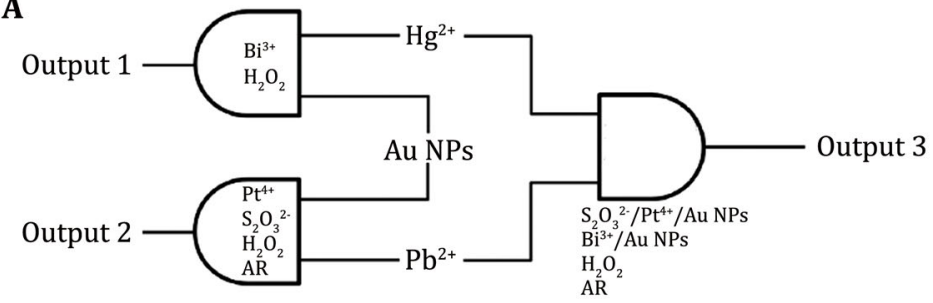

B

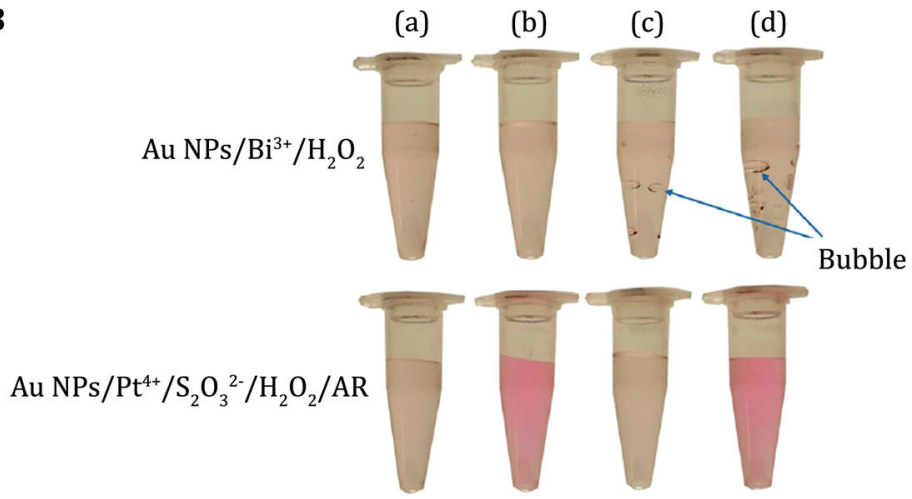

C

\begin{tabular}{ccccc}
\hline $\mathrm{Pb}^{2+}$ & $\mathrm{Hg}^{2+}$ & $\begin{array}{c}\text { Output 1 } \\
\mathrm{O}_{2} \text { bubble }\end{array}$ & $\begin{array}{c}\text { Output 2 } \\
\text { reddish resorufin }\end{array}$ & $\begin{array}{c}\text { Output 3 } \\
\mathrm{O}_{2} \text { bubble and } \\
\text { reddish resorufin }\end{array}$ \\
\hline 0 & 0 & 0 & 0 & 0 \\
1 & 0 & 0 & 1 & 0 \\
0 & 1 & 1 & 0 & 0 \\
1 & 1 & 1 & 1 & 1 \\
\hline
\end{tabular}

contradictory. Nucleic acids could enhance the activity of nanozymes since DNA absorbed on the surface of nanozyme could increase substrates binding through electrostatic interaction (Liu and Liu 2015). In the other case, nucleic acids suppressed the catalytic reaction by absorbing on the surface of nanozyme to block the active sites (Zheng et al. 2011). Although the mechanism is not yet comprehended, nucleic acid-regulated nanozyme-based logic gates were constructed.

AuNPs can mimic the catalytic activity of glucose oxidase (GOx). Nucleic acids absorbed on the surface of AuNPs change the surface properties, thus affecting the catalytic activity. Zhan et al. investigated the influence of $\mathrm{pH}$-sensitive nucleic acid strands on AuNPs and demonstrated $\mathrm{pH}$-induced conformational change of DNA strands endows AuNPs with different catalytic efficiency (Zhan et al. 2014). Using $\mathrm{pH}$ and different DNA strands as inputs, a series of logic gates (AND, XNOR, and NOT) were designed.

Nucleic acids can act as a linker to control assembly of nanoparticles through programmed Watson-Crick base pairing. Taking advantage of the properties of nucleic acid, Lin et al. developed a nanozyme-based bio-barcode fluorescence assay (Lin et al. 2017). The complementary DNA sequence of HIV (CHIV) was split into two segments, CHIV1 and CHIV2. Then they were modified on the surface of bimetallic nanoparticle PtAuNPs and magnetic nanoparticles (MNPs), respectively. Upon addition of HIV DNA, PtAuNPs and MNPs would be linked together via hybridization of HIV DNA with the two segments. After magnetic separation, amplex red (AR) and $\mathrm{H}_{2} \mathrm{O}_{2}$ were added. PtAuNPs presented peroxidase-like activity and could catalyze nonfluorescent substrate AR into fluorescence product. The concentration of HIV DNA could be quantified by measuring the change of fluorescence induced by the catalytic reaction. Similarly, HCV DNA could also be detected based on the same principle. Using HIV and HCV DNA as inputs, an OR logic gate was constructed (Lin et al. 2017). Furthermore, cascaded INHIBIT-OR logic gate was integrated with a biosensor to distinguish individual HIV and HCV genes in mixture. The CHIV as mask agent could depress signal change caused by HIV DNA. An INHIBIT gate could be constructed using CHIV 
and HIV DNA as inputs. By further integrating with the OR gate, HIV and HCV DNA could be distinguished.

Nucleic acid can also drive assembly of nanoparticles through specific recognition. Based on the high affinity between aptamer and protein, Lin et al. used protein-mediated aggregation of DNA-absorbed hemingraphene nanosheet to build colorimetric protein assay and logic gates (Fig. 3) (Lin et al. 2014). Hemin-graphene (HG) hybrid nanosheet possesses intrinsic peroxidase-like catalytic activity. The self-assembly of the hybrid nanosheet can cause precipitation after centrifugation. The supernatant presented low catalytic activity due to the low amount of the hybrid nanosheet. The interaction between proteins and aptamers absorbed on the surface of hemin-graphene nanosheet led to the cross-linking aggregation, thus changing the colorimetric signal of the supernatant. Thrombin and PDGF$\mathrm{BB}$ as model analytes could be detected with high sensitivity. Furthermore, using thrombin and PDGF-BB as inputs and aptamer-absorbed HG as logic unit, an OR

A

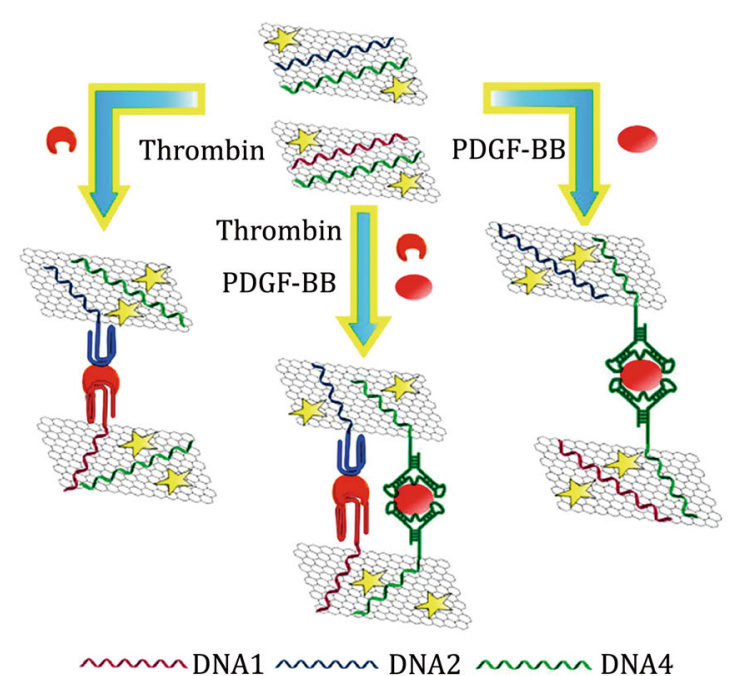

\begin{tabular}{|c|c|c|}
\hline $\begin{array}{c}\text { Input } \\
\text { Thrombin }\end{array}$ & $\begin{array}{c}\text { Input } \\
\text { PDGF-BB }\end{array}$ & $\begin{array}{c}\text { OR } \\
\text { Output }\end{array}$ \\
\hline 0 & 0 & 0 \\
\hline 1 & 0 & 1 \\
\hline 0 & 1 & 1 \\
\hline 1 & 1 & 1 \\
\hline
\end{tabular}

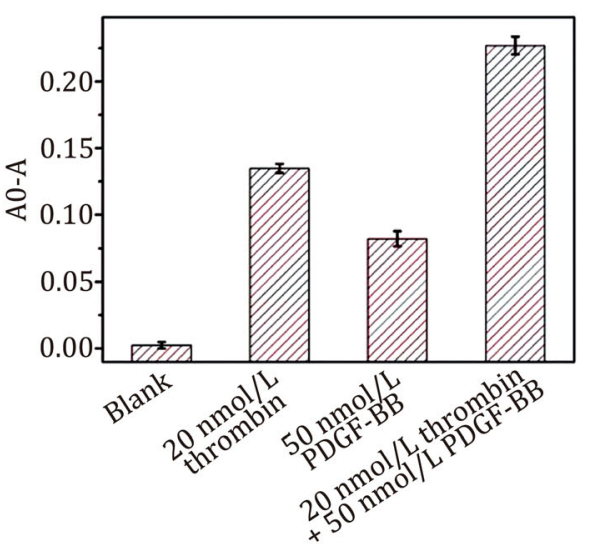

B

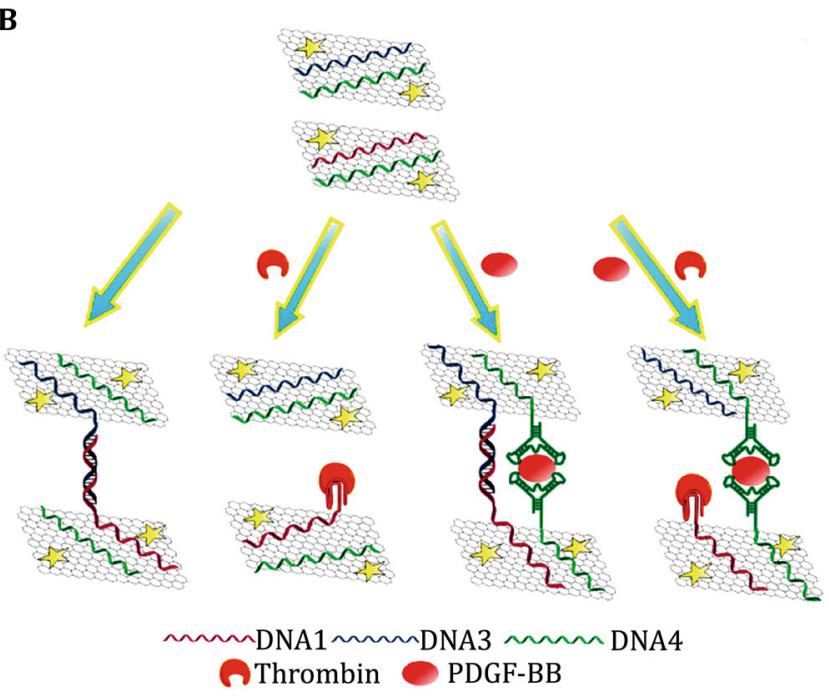

\begin{tabular}{|c|c|c|}
\hline $\begin{array}{c}\text { Input } \\
\text { Thrombin }\end{array}$ & $\begin{array}{c}\text { Input } \\
\text { PDGF-BB }\end{array}$ & $\begin{array}{c}\text { Inhibit } \\
\text { Output }\end{array}$ \\
\hline 0 & 0 & 0 \\
\hline 1 & 0 & 1 \\
\hline 0 & 1 & 0 \\
\hline 1 & 1 & 0 \\
\hline
\end{tabular}

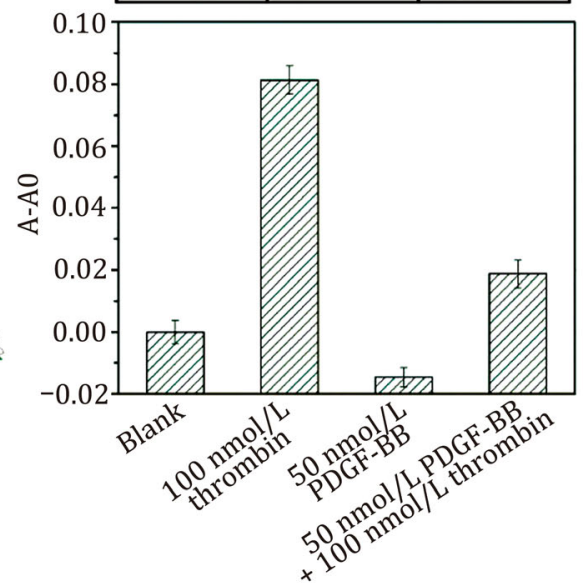

Fig. 3 OR (A) and INHIBIT (B) gates using protein-mediated aggregation of DNA-absorbed hemin-graphene nanosheet. Reproduced with permission of American Chemical Society (Lin et al. 2014) 
gate could be constructed. In the presence of thrombin, PDGF-BB, and their mixture, sandwich structure of HG/ DNA/protein could be formed through specific binding between proteins and their aptamers, leading to reduction of catalytic activity of centrifugal supernatant. By changing DNA sequence, an INHIBIT operation could be realized. Two DNA strands absorbed on the surface of HG could hybrid to cause aggregation of HG. Since one of them was aptamer of thrombin, the addition of thrombin could inhibit their hybridization and aggregation of HG. However, PDGF-BB not only did not disturb the hybridization, but also caused formation of sandwich structure of HG/aptamer/PDGF-BB. Adding both thrombin and PDGF-BB still resulted in aggregation of HG. The output signals corresponded to an INHIBIT gate.

Glutathione (GSH), tripeptide containing a thiol group, plays crucial roles in several biological processes. GSH could change the catalytic activity of nanozyme by reacting with nanozyme. Manganese dioxide nanosheet $\left(\mathrm{MnO}_{2} \mathrm{NS}\right)$ was regarded as an oxidase mimic and fluorescent Scopoletin (SC) and nonfluorescent AR could serve as the substrates of $\mathrm{MnO}_{2} \mathrm{NS}$. Fan et al. found $\mathrm{MnO}_{2}$ NS could quench the fluorescence of SC and enhance the fluorescence of AR through oxidation reaction (Fan et al. 2017). However, GSH could reduce $\mathrm{MnO}_{2}$ to $\mathrm{Mn}^{2+}$, leading to the loss of oxidase-like activity. The fluorescence of SC increased while the fluorescence of AR decreased. Based on the dual-signal response, a ratiometric sensor for detection of GSH was constructed. The output signals corresponded to an INHIBIT gate cascade with a 1 to 2 decoder (Fig. 4).
Some biomolecules could interact with composition of nanozymes and block their catalytic activity. Copper (II)-based metal-organic frameworks (Cu-MOFs) showed peroxidase-like activity. The catalytic activity of $\mathrm{Cu}-\mathrm{MOF}$ was greatly inhibited by pyrophosphate (PPi) due to the ultrastrong binding between $\mathrm{Cu}^{2+}$ and PPi. Alkaline phosphatase (ALP) could hydrolyze PPi into orthophosphates (Pi), which have low affinity to $\mathrm{Cu}^{2+}$. This led to the recovery of catalytic activity of Cu-MOFs, allowing visual analysis of ALP. Taking advantage of the activity change induced by PPi and ALP, Wang et al. constructed an IMPLICATION logic gate (Wang et al. 2018a). Based on the similar principle, Chen et al. used histidine-protected gold nanoclusters (His-AuNCs) with peroxidase-like activity constructed colorimetric sensor for phosphate-containing metabolites and logic gate (Chen et al. 2019). Besides PPi, other phosphatecontaining metabolites including adenosine triphosphate (ATP) and adenosine diphosphate (ADP) could hamper the catalytic activity of His-AuNCs and be hydrolyzed by ALP. Using the three phosphatecontaining metabolites as inputs and His-AuNCs as logic unit, a NOR gate was built. By further adding ALP as input, a NOR+IMPLICATION gate was constructed.

$\mathrm{NH}_{2}$-MIL-101 MOFs as a peroxidase-like nanozyme catalyze oxidation of o-phenylenediamine (OPD) by $\mathrm{H}_{2} \mathrm{O}_{2}$ to generate fluorescent 2,3-diaminophenazine. In the catalytic reaction, the inherent fluorescence emission at $456 \mathrm{~nm}\left(\mathrm{~F}_{456}\right)$ of $\mathrm{NH}_{2}$-MIL-101 MOFs was quenched, while the fluorescence of catalytic product 2,3-diaminophenazine at $556 \mathrm{~nm}\left(\mathrm{~F}_{556}\right)$ increased. The catalytic activity could be inhibited by PPi through binding of PPi and Fe center, and then restored by acid
Fig. 4 Scheme of the cascade logic circuit (INHIBIT-1 to 2 Decoder). Reproduced with permission of American Chemical Society (Fan et al. 2017)

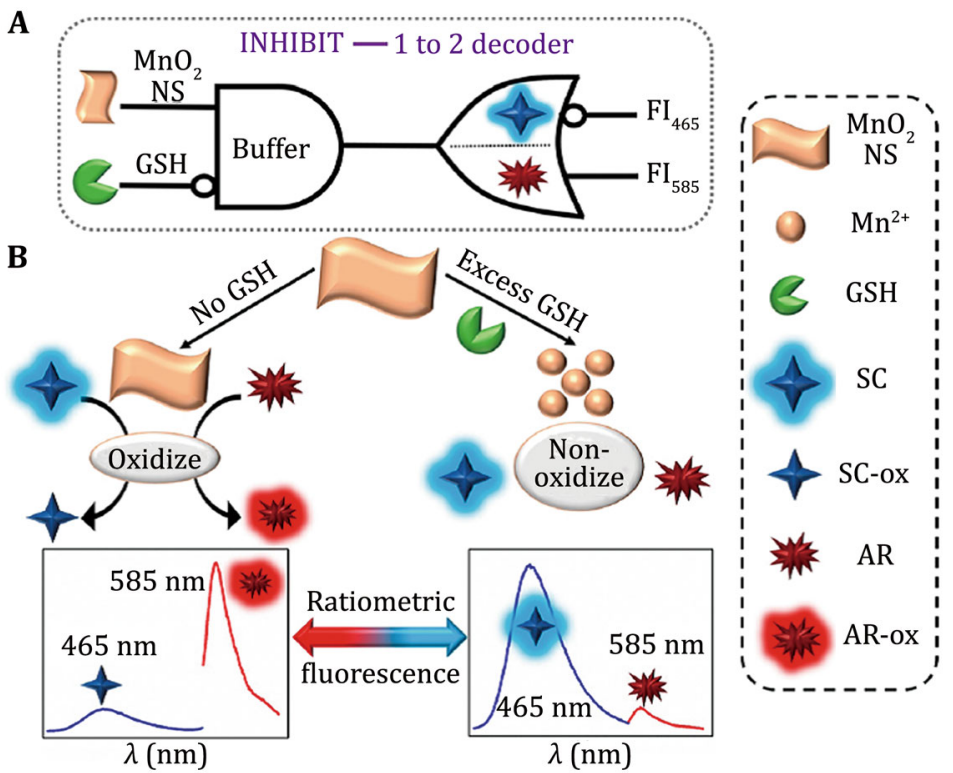


phosphatase (ACP). Based on the PPi/ACP-mediated fluorescence changes at different wavelengths, Li et al. constructed a ratiometric fluorescence sensor for ACP and an INHIBIT gate cascade with 1-2 decoder (Li et al. 2019).

\section{Light}

Light as modulator of nanozyme attracts great attention owing to its high temporal and spatial precision, and ease in controlling the intensity and wavelength (Huang et al. 2019; Wu et al. 2019b). Different from the most nanozymes who showed the "on" state under normal conditions, photo-responsive nanozymes presented catalytic activity in the presence of light. For example, upon visible light illumination, 9-mesityl-10methylacridinium ion $\left(\mathrm{Acr}^{+}\right.$-Mes) presented oxidaselike activity for catalytic oxidation of $3,3^{\prime}, 5,5^{\prime}$ tetramethylbenzidine (TMB). Du et al. constructed visual logic gates (AND, INH, and NOR) based on the catalytic reaction (Fig. 5) (Du et al. 2018). An AND logic operation could be performed using TMB as logic unit and $\mathrm{Acr}^{+}$-Mes and light illumination as inputs. L-cysteine and L-glutathione could restrain the photo-oxidation of TMB by $\mathrm{Acr}^{+}$-Mes. Using TMB and light illumination as logic unit and L-cysteine and $\mathrm{Acr}^{+}$-Mes as inputs, an INH gate was constructed. Using TMB, light illumination and $\mathrm{Acr}^{+}$-Mes as logic unit and L-cysteine and L-glutathione as inputs, an OR gate was constructed. The system could be used for colorimetric detection of L-cysteine and L-glutathione.

\section{pH}

One nanozyme can show different types or catalytic efficiency at different $\mathrm{pH}$. For example, $\mathrm{Fe}_{3} \mathrm{O}_{4}$ nanoparticles exhibit peroxidase-like activity at acidic $\mathrm{pH}$ while exhibit catalase-like activity under neutral environment. Also, the oxidase-like activities of $\mathrm{MnO}_{2}$ nanosheets are $\mathrm{pH}$ switchable and reversible. At acidic condition, $\mathrm{MnO}_{2}$ nanosheets show oxidase-like activity. When $\mathrm{pH}$ becomes alkaline, the activity decreases due to the transformation into hexagonal $\mathrm{Na}_{4} \mathrm{MnO}_{4}$. Based on the phenomenon, Zhao et al. constructed a three-input molecular majority
Fig. 5 Scheme, output results, and truth table of logic gates based on light-activatable $\mathrm{Acr}^{+}$-Mes with an oxidase-like activity. Reproduced with permission of American Chemical Society (Du et al. 2018)
A

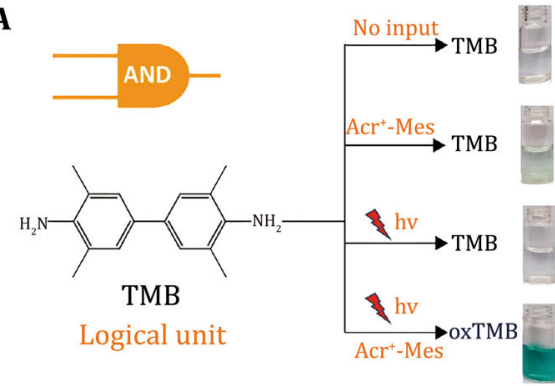

B

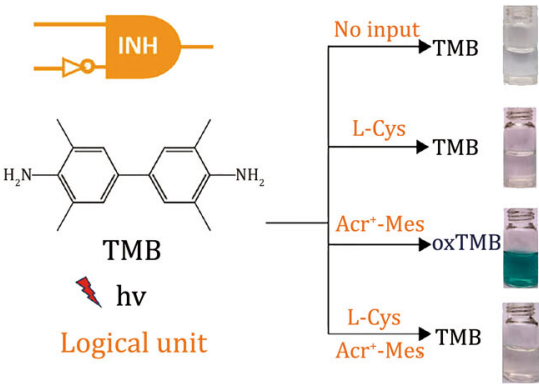

C

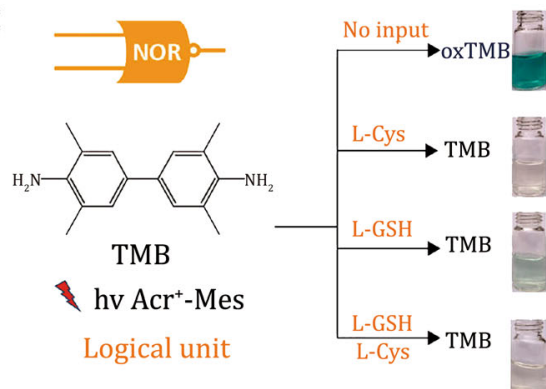

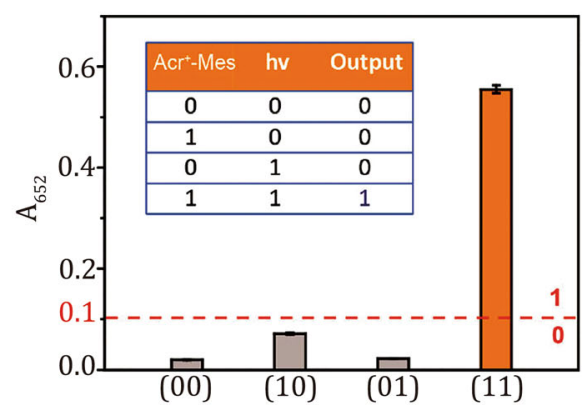
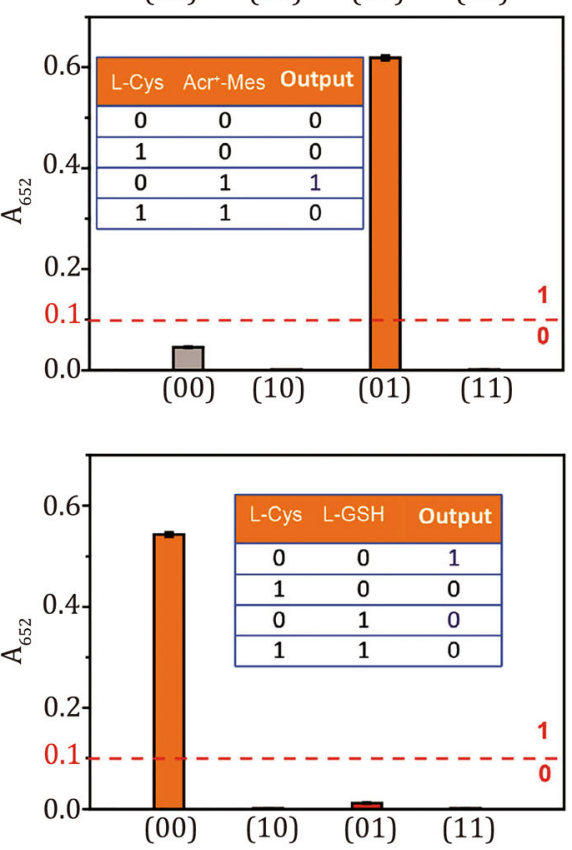
logic gate with a single-vote veto function (Fig. 6). The alkaline TMB solution served as the logic unit, and the $\mathrm{MnO}_{2}$ nanosheets, $\mathrm{H}^{+}$, and $\mathrm{H}_{2} \mathrm{O}_{2}$ served as inputs. Only when two or more inputs were added, the oxidation of TMB to oxTMB occurred, which corresponded to a threeinput major logic function. However, in the strong basic condition, the oxidation of TMB was inhibited since the oxidation capacity of $\mathrm{H}_{2} \mathrm{O}_{2}$ and oxidase-like activity of the $\mathrm{MnO}_{2}$ nanosheets remarkably reduced. Thus, $\mathrm{NaOH}$ as the other input could perform a single-vote veto function. In addition, using $\mathrm{H}^{+}$and TMB as logic unit, and $\mathrm{H}_{2} \mathrm{O}_{2}$, $\mathrm{MnO}_{2}$, and $\mathrm{NaOH}$ as inputs, an OR-INH cascade logic circuit could be realized (Zhao et al. 2018).

\section{Multiplex factors}

In general, definitive logic unit and inputs output fixed logic signal. It is difficult and significant to realize logic conversion of sole logic system. Inspired from nature in which organisms can be programmed into different forms in different environment, Han et al. realized the transformation of logic functions using definitive logic unit and inputs (Fig. 7) (Han et al. 2018). The metal ions could alter the catalase-like activity of $\mathrm{Co}_{3} \mathrm{O}_{4}$ nanoparticles in type- and pH-dependent manners. Only by changing $\mathrm{pH}$ of the logic systems, switchable logic operations could be realized. For the system using $\mathrm{Co}_{3} \mathrm{O}_{4}$ as logic unit and $\mathrm{Hg}^{2+} / \mathrm{Fe}^{3+}$ as inputs, an OR gate was constructed at $\mathrm{pH}$ 10.0. When the $\mathrm{pH}$ was changed to 7.0, the system performed an INHIBIT function. Using $\mathrm{Ca}^{2+}$ and $\mathrm{Mn}^{2+}$ as inputs, INHIBIT and AND gates were obtained at $\mathrm{pH} 10.0$ and $\mathrm{pH}$ 7.0, respectively.

\section{SUMMARY AND PERSPECTIVES}

In summary, recent development of nanozyme-based logic gates was summarized. Despite this valuable
Fig. 6 A Scheme of the threeinput majority logic gate based on pH-modulated oxidase-like activity of $\mathrm{MnO}_{2}$ nanosheets. B UV-vis absorption spectra (a), photographs (b), absorbance intensities at $652 \mathrm{~nm}$ (c), and truth table (d) of the three-input majority logic gate with different combinations of the inputs. Input 1 , input 2 , and input 3 were $\mathrm{H}_{2} \mathrm{O}_{2}, \mathrm{MnO}_{2}$, and $\mathrm{H}^{+}$, respectively. The absence and presence of the inputs represented 0 and 1 , respectively. I-VIII: $(0,0,0)$, $(1,0,0),(0,1,0),(0,0,1),(1$, $0,1),(1,1,0),(0,1,1),(1,1$, 1); IX: $\mathrm{NaOH}$ and $(1,1,1)$. Reproduced with permission of Royal Society of Chemistry (Zhao et al. 2018)
A
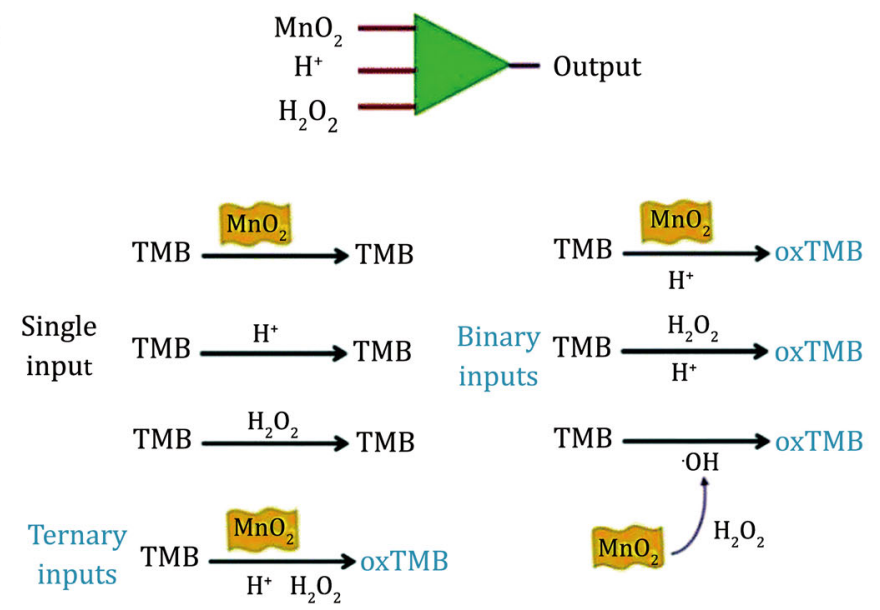

B
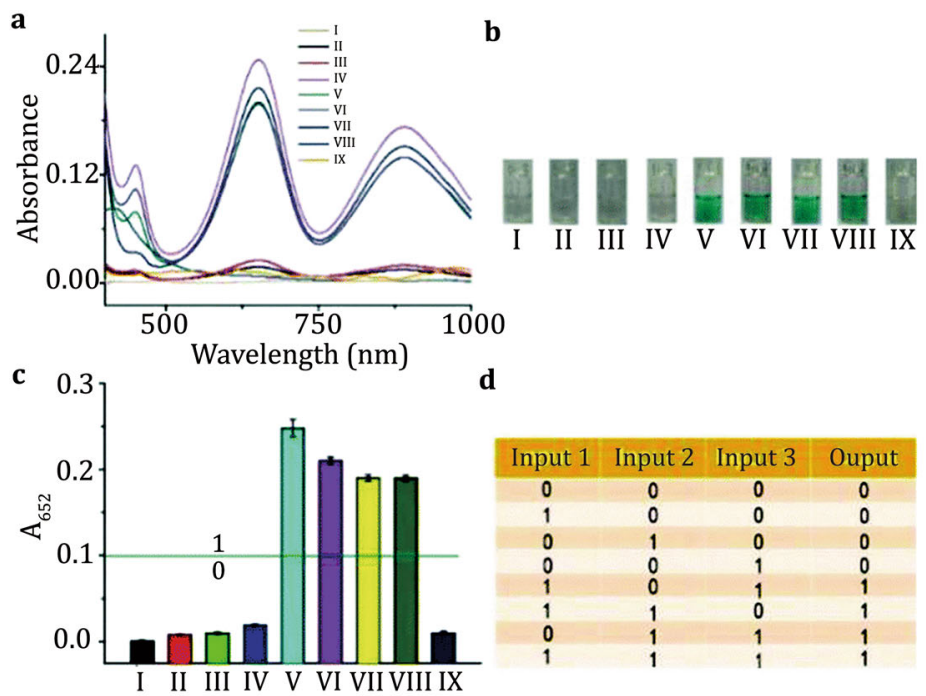

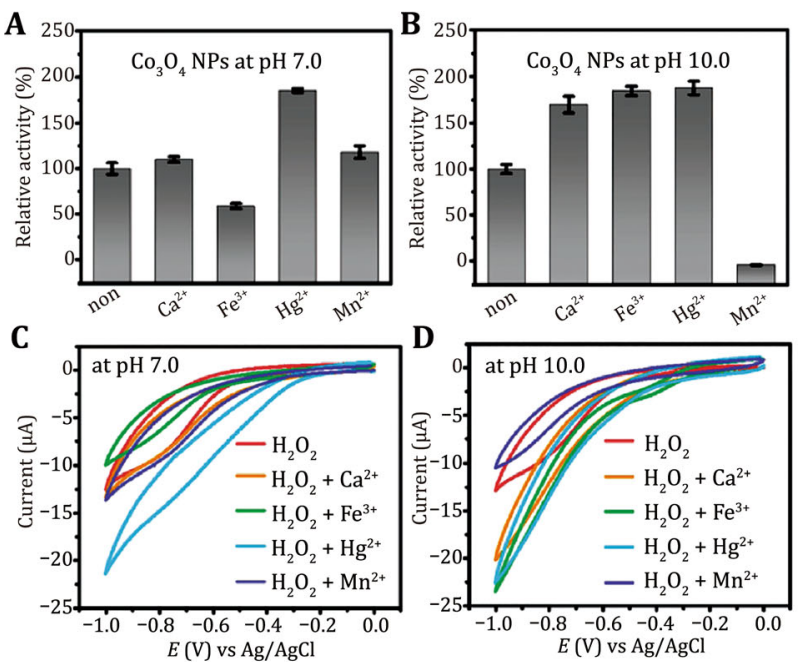

$\mathbf{E}$

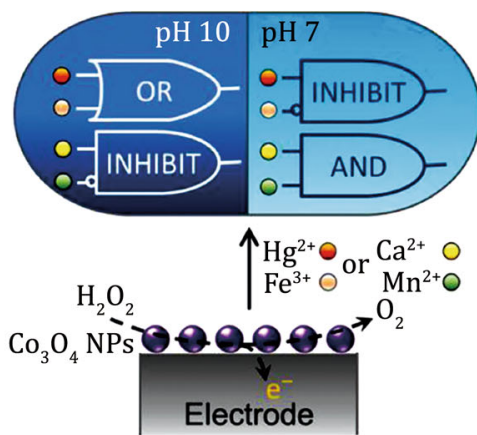

$\mathbf{F}$
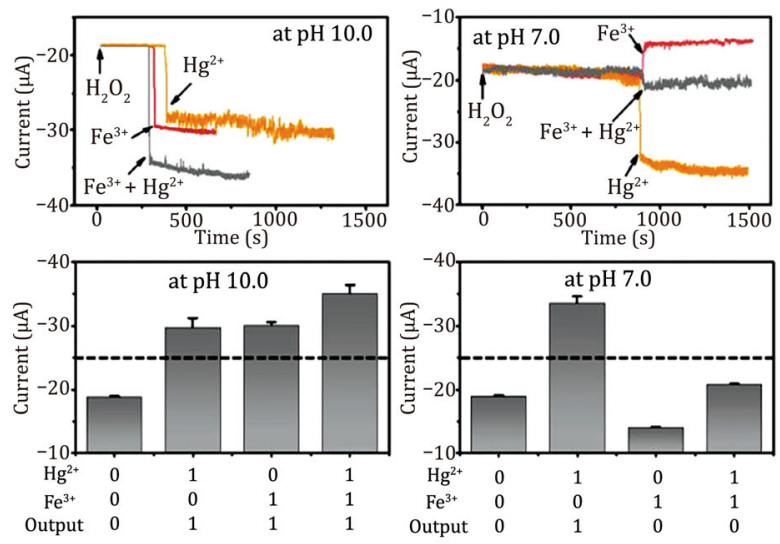

G
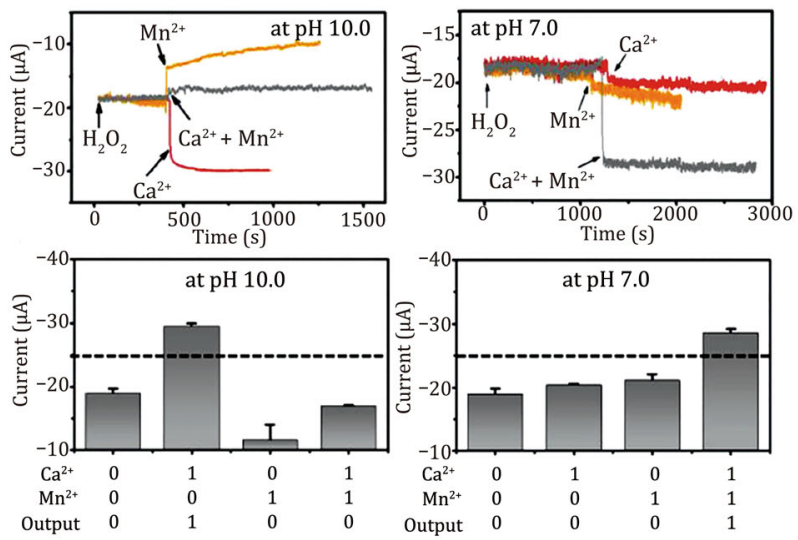

Fig. 7 Influence of different metal ions on the catalase-like activity (A, B) and electrocatalytic activity $(\mathbf{C}, \mathbf{D})$ of $\mathrm{Co}_{3} \mathrm{O}_{4}$ nanoparticles at $\mathrm{pH}$ 7.0 and $\mathrm{pH}$ 10.0. E Scheme of $\mathrm{pH}$-switchable logic conversation. F OR/INHIBIT-switchable logic gate. G INHIBIT/AND-switchable logic gate. Reproduced with permission of Wiley-VCH (Han et al. 2018)

progress, nanozyme-based logic gates remain in its early stage. Some challenges still exist in this field.

(1) The naonzymes used for construction of logic gates are mainly mimics of oxidoreductase, such as peroxidase, oxidase, and catalase. This is limited by the type of nanozymes themselves. With the development of new nanozymes which can catalyze other types of reaction beyond redox reactions, the range of selection will expand. Moreover, nanozymes can be combined with natural enzymes or other chemical reactions to achieve logic functions.

(2) Most of the current nanozyme-based logic gates could only implement simple logic operations, such as AND, OR, and INHIBIT. Constructing complex logic systems to realize advanced information processing is less explored. This can be attributed to the high complexity of circuit design. For example, different nanozymes have their own optimal reaction conditions. When they are in a uniform reaction system, they may show low catalytic efficiency and unsatisfactory stability, thus limiting the realization of logic functions.

(3) Nanozymes could interact with a series of biomolecules, including proteins, nucleic acids, peptides, and even cells (Qiu et al. 2018; Wang et al. 2018c). The nonspecific binding of these biomolecules could interfere with the catalytic activity of nanozymes. Therefore, it is necessary to consider this factor in the design of logic gates integrated with sensors and diagnostic reagents.

(4) Some external substances could regulate the catalytic activity of nanozymes. Although they acted as inputs to construct logic gates, the mechanism of their regulation effect on nanozyme activity has not been fully understood.

(5) The reset capability is a key factor for the reusability of logic gates. However, it is difficult to realize the reset of these nanozyme-based logic systems to the original state since the inputs are hard to be separated from the reaction solution. Moreover, the accumulation of by-product may affect the performance of logic gates. Therefore, 
design of resettable nanozyme-based logic systems is a crucial study for practical applications.

(6) An important goal of constructing molecular logic systems is the ultimate translation for practical applications. However, due to limited complexity of molecular logic gates, it is difficult to compete with electronic computers. We had to think about the issue with a different view. The molecular logic gates can simplify the bioinformation and output the diagnosis or detection result as "0" or " 1 ," which means the absence or presence of disease and analytes. This provides another avenue for applications of molecular logic gates. Several molecular logic gates have been integrated with various functions, such as drug delivery/release, bioimaging, disease diagnosis, regulating the expression of specific disease genes, and biosensing $(\mathrm{Pu}$ et al. 2014a; Ran et al. 2018). However, most nanozymebased logic gates were only integrated with biosensors. Nanozymes have been used for cancer treatment, inflammation elimination, and antibacterial therapy based on their catalytic properties in response to the physiological environment (Huang et al. 2016; Qiu et al. 2020; Wang et al. 2018d). The logic gates coupling with these biological applications will offer future intelligent diagnosis and therapy.

(7) The current nanozyme-based logic functions were performed in solution. Absorbance and fluorescence signals were used as outputs. The characteristics of nanomaterials enable them to be modified on the interface to realize catalytic reactions, which can offer transduction of the biochemical output into electronic or other signals. This may provide an opportunity for the fabrication of portable logic devices.

Nonetheless, the advantages of nanozymes still offer tremendous opportunities for biologic information processing. With further development of nanomaterials with enzyme-like activity, more sophisticated and useful logic systems will be designed and built.

Acknowledgements Studies in our laboratory were aided by the National Key R\&D Program of China (2019YFA0709202), National Natural Science Foundation of China (21533008, 21871249, 91856205, 21673223, 21977091 and 21820102009), and Key Program of Frontier of Sciences (CAS QYZDJ-SSW-SLH052).

\section{Compliance with Ethical Standards}

Conflict of interest Fang $\mathrm{Pu}$, Jinsong Ren, and Xiaogang $\mathrm{Qu}$ declare that they have no conflict of interest.
Human and animal rights and informed consent This article does not contain any studies with human or animal subjects performed by any of the authors.

Open Access This article is licensed under a Creative Commons Attribution 4.0 International License, which permits use, sharing, adaptation, distribution and reproduction in any medium or format, as long as you give appropriate credit to the original author(s) and the source, provide a link to the Creative Commons licence, and indicate if changes were made. The images or other third party material in this article are included in the article's Creative Commons licence, unless indicated otherwise in a credit line to the material. If material is not included in the article's Creative Commons licence and your intended use is not permitted by statutory regulation or exceeds the permitted use, you will need to obtain permission directly from the copyright holder. To view a copy of this licence, visit http://creativecommons.org/ licenses/by/4.0/.

\section{References}

Andre R, Natalio F, Humanes M, Leppin J, Heinze K, Wever R, Schroder HC, Muller WEG, Tremel W (2011) $\mathrm{V}_{2} \mathrm{O}_{5}$ nanowires with an intrinsic peroxidase-like activity. Adv Funct Mater 21:501-509

Andreasson J, Pischel U (2010) Smart molecules at workmimicking advanced logic operations. Chem Soc Rev 39:174-188

Chen C, Zhao D, Jiang Y, Ni P, Zhang C, Wang B, Yang F, Lu Y, Sun J (2019) Logically regulating peroxidase-like activity of gold nanoclusters for sensing phosphate-containing metabolites and alkaline phosphatase activity. Anal Chem 91:15017-15024

de Silva AP, McClenaghan ND (2004) Molecular-scale logic gates. Chem Eur J 10:574-586

Du J, Wang J, Huang W, Deng Y, He Y (2018) Visible lightactivatable oxidase mimic of 9-mesityl-10-methylacridinium ion for colorimetric detection of biothiols and logic operations. Anal Chem 90:9959-9965

Erbas-Cakmak S, Kolemen S, Sedgwick AC, Gunnlaugsson T, James TD, Yoon J, Akkaya EU (2018) Molecular logic gates: the past, present and future. Chem Soc Rev 47:2228-2248

Fan D, Shang C, Gu W, Wang E, Dong S (2017) Introducing ratiometric fluorescence to $\mathrm{MnO}_{2}$ nanosheet-based biosensing: a simple, label-free ratiometric fluorescent sensor programmed by cascade logic circuit for ultrasensitive GSH detection. ACS Appl Mater Interfaces 9:25870-25877

Gao L, Zhuang J, Nie L, Zhang J, Zhang Y, Gu N, Wang T, Feng J, Yang D, Perrett S, Yan X (2007) Intrinsic peroxidase-like activity of ferromagnetic nanoparticles. Nat Nanotechnol 2:577-583

Han L, Zhang H, Li F (2018) Bioinspired nanozymes with $\mathrm{pH}$-independent and metal ions-controllable activity: fieldprogrammable logic conversion of sole logic gate system. Part Part Syst Charact 35(9):1800207. https://doi.org/10. 1002/ppsc.201800207

Huang Y, Liu Z, Liu C, Ju E, Zhang Y, Ren J, Qu X (2016) Selfassembly of multi-nanozymes to mimic an intracellular antioxidant defense system. Angew Chem Int Ed 55:6646-6650

Huang Y, Pu F, Ren J, Qu X (2017) Artificial enzyme-based logic operations to mimic an intracellular enzyme-participated redox balance system. Chem Eur J 23:9156-9161 
Huang Y, Lin Y, Pu F, Ren J, Qu X (2018) The current progress of nanozymes in disease treatments. Prog Biochem Biophys 45:256-267

Huang Y, Ren J, Qu X (2019) Nanozymes: classification, catalytic mechanisms, activity regulation, and applications. Chem Rev 119:4357-4412

Katz E, Privman V (2010) Enzyme-based logic systems for information processing. Chem Soc Rev 39:1835-1857

Li S, Hu X, Chen Q, Zhang X, Chai H, Huang Y (2019) Introducing bifunctional metal-organic frameworks to the construction of a novel ratiometric fluorescence sensor for screening acid phosphatase activity. Biosens Bioelectron 137:133-139

Lien CW, Chen YC, Chang HT, Huang CC (2013) Logical regulation of the enzyme-like activity of gold nanoparticles by using heavy metal ions. Nanoscale 5:8227-8234

Lien CW, Tseng YT, Huang CC, Chang HT (2014) Logic control of enzyme-like gold nanoparticles for selective detection of lead and mercury ions. Anal Chem 86:2065-2072

Lin Y, Xu C, Ren J, Qu X (2012) Using thermally regenerable cerium oxide nanoparticles in biocomputing to perform label-free, resettable, and colorimetric logic operations. Angew Chem Int Ed 51:12579-12583

Lin B, Sun QQ Liu K, Lu DQ Fu Y, Xu ZA, Zhang W (2014) Labelfree colorimetric protein assay and logic gates design based on the self-assembly of hemin-graphene hybrid nanosheet. Langmuir 30:2144-2151

Lin XD, Liu YQ, Tao ZH, Gao JT, Deng JK, Yin JJ, Wang S (2017) Nanozyme-based bio-barcode assay for high sensitive and logic-controlled specific detection of multiple DNAs. Biosens Bioelectron 94:471-477

Liu B, Liu J (2015) Accelerating peroxidase mimicking nanozymes using DNA. Nanoscale 7:13831-13835

$\mathrm{Mu}$ J, Zhang $\mathrm{L}$, Zhao $\mathrm{M}$, Wang $\mathrm{Y}$ (2013) $\mathrm{Co}_{3} \mathrm{O}_{4}$ nanoparticles as an efficient catalase mimic: properties, mechanism and its electrocatalytic sensing application for hydrogen peroxide. J Mol Catal A 378:30-37

Pu F, Ju E, Ren J, Qu X (2014a) Multiconfigurable logic gates based on fluorescence switching in adaptive coordination polymer nanoparticles. Adv Mater 26:1111-1117

Pu F, Ren J, Qu X (2014b) Nucleic acids and smart materials: advanced building blocks for logic systems. Adv Mater 26:5742-5757

Qiu H, Pu F, Ran X, Liu C, Ren J, Qu X (2018) Nanozyme as artificial receptor with multiple readouts for pattern recognition. Anal Chem 90:11775-11779

Qiu H, Pu F, Liu Z, Liu X, Dong K, Liu C, Ren J, Qu X (2020) Hydrogel-based artificial enzyme for combating bacteria and accelerating wound healing. Nano Res 13:496-502

Ran X, Wang Z, Ju E, Pu F, Song Y, Qu X, Ren J (2018) An intelligent 1:2 demultiplexer as an intracellular theranostic device based on DNA/Ag cluster-gated nanovehicles. Nanotechnology 29(6):065501. https://doi.org/10.1088/1361-6528/aaa09a
Sharma V, Mobin SM (2017) Cytocompatible peroxidase mimic $\mathrm{CuO}$ :graphene nanosphere composite as colorimetric dual sensor for hydrogen peroxide and cholesterol with its logic gate implementation. Sens Actuators B 240:338-348

Song Y, Qu K, Zhao C, Ren J, Qu X (2010) Graphene oxide: intrinsic peroxidase catalytic activity and its application to glucose detection. Adv Mater 22:2206-2210

Wang XY, Hu YH, Wei H (2016) Nanozymes in bionanotechnology: from sensing to therapeutics and beyond. Inorg Chem Front 3:41-60

Wang CH, Gao J, Cao YL, Tan HL (2018a) Colorimetric logic gate for alkaline phosphatase based on copper (II)-based metalorganic frameworks with peroxidase-like activity. Anal Chim Acta 1004:74-81

Wang H, Li P, Yu D, Zhang Y, Wang Z, Liu C, Qiu H, Liu Z, Ren J, Qu X (2018b) Unraveling the enzymatic activity of oxygenated carbon nanotubes and their application in the treatment of bacterial infections. Nano Lett 18:3344-3351

Wang XY, Qin L, Zhou M, Lou ZP, Wei H (2018c) Nanozyme sensor arrays for detecting versatile analytes from small molecules to proteins and cells. Anal Chem 90:11696-11702

Wang Z, Zhang Y, Ju E, Liu Z, Cao F, Chen Z, Ren J, Qu X (2018d) Biomimetic nanoflowers by self-assembly of nanozymes to induce intracellular oxidative damage against hypoxic tumors. Nat Commun 9(1):3334. https://doi.org/10.1038/ s41467-018-05798-x

Wei H, Wang E (2013) Nanomaterials with enzyme-like characteristics (nanozymes): next-generation artificial enzymes. Chem Soc Rev 42:6060-6093

Wu J, Wang X, Wang Q, Lou Z, Li S, Zhu Y, Qin L, Wei H (2019a) Nanomaterials with enzyme-like characteristics (nanozymes): next-generation artificial enzymes (II). Chem Soc Rev 48:1004-1076

Wu SH, Zhang JY, Wu P (2019b) Photo-modulated nanozymes for biosensing and biomedical applications. Anal Methods 11:5081-5088

Zhan P, Wang J, Wang ZG, Ding B (2014) Engineering the pHresponsive catalytic behavior of AuNPs by DNA. Small 10:399-406

Zhang H, Liang X, Han L, Li F (2018) "Non-naked" gold with glucose oxidase-like activity: a nanozyme for tandem catalysis. Small 14:1803256. https://doi.org/10.1002/smll. 201803256

Zhao M, Tao Y, Huang W, He Y (2018) Reversible pH switchable oxidase-like activities of $\mathrm{MnO}_{2}$ nanosheets for a visual molecular majority logic gate. Phys Chem Chem Phys 20:28644-28648

Zheng X, Liu Q Jing C, Li Y, Li D, Luo W, Wen Y, He Y, Huang Q Long YT, Fan C (2011) Catalytic gold nanoparticles for nanoplasmonic detection of DNA hybridization. Angew Chem Int Ed 50:11994-11998 